\title{
A catastrophic nightmare of the interventional cardiologist: Iatrogenic left main artery dissection and longitudinal stent deformation
}

\author{
Oguz Yıldırım, Murat Gul, Sinan Inci*, Halil Aktaş, Pınar Dogan, Abdulkadir Bozkır, \\ İbrahim İzgü , Recep Karataş
}

Department of Cardiology, Aksaray Training and Research Hospital, Aksaray University, Aksaray, Turkey.

\begin{abstract}
Summary Iatrogenic left main coronary artery dissection is a rare but potentially life-threatening complication of invasive coronary procedures. The newer generation drug eluting stents have shown a greater safety and efficacy compared to first generation drug eluting stents. We report a 60-year-old woman with iatrogenic left main coronary artery dissection who failed bailout stenting and underwent coronary artery bypass grafting. The strategy for managing left main coronary artery dissection is variable and depends upon the mechanism, the comorbidities of the patient and degree of hemodynamic stability. Longitudinal stent deformation is a rarely encountered complication but can be seen in complex lesions such as ostial, bifurcation and left main coronary artery lesions. The interventionists must be aware of this complication.
\end{abstract}

Keywords: Left main coronary artery dissection, longitudinal stent deformation, drug eluting stents

\section{Introduction}

Iatrogenic left main coronary artery (LMCA) dissection is a rare but potentially life-threatening complication of invasive coronary procedures with a reported incidence of less than $0.1 \%$ (1). Timely recognition of the dissection and construction of a proper treatment plan based on the type of the dissection and the clinical status of the patient is needed to overcome this potentially fatal complication. Treatment consists of conservative therapy, salvage percutaneous coronary intervention (PCI) or urgent coronary artery bypass graft (CABG) surgery.

The newer generation drug eluting stents (DES) have shown greater safety and efficacy compared to first generation DES, because of thinner struts, nondurable polymers and coating with better anti-proliferative drugs $(2,3)$. Though, the current stent design of thin struts and cobalt alloy has improved the technical performance

Released online in J-STAGE as advance publication August 28, 2018.

*Address correspondence to:

Dr. Sinan Inci, Department of Cardiology, Aksaray Training and Research Hospital, Aksaray University, Aksaray 06100, Turkey.

E-mail: doktorsinaninci@gmail.com of various stents in terms of tractability, conformability and flexibility; and their poor longitudinal axial strength makes them susceptible to longitudinal stent deformation (LSD) (4). Herein we report a case of iatrogenic LMCA dissection that failed bailout stenting and underwent CABG.

\section{Case Report}

A 60-year-old female patient with a history of hyperlipidemia and smoking was referred to the cardiology clinic following complaints of chest pain and dyspnea for the last 24 hours. It was also noted that she had been suffering from a cough for the past one week as a result of an upper respiratory tract infection. Physical examination was unremarkable, with BP 136/88 mmHg and HR 95 beats per minute, regular. Electrocardiography revealed sinus rhythm with new T-wave inversions in leads V1-V4. The echocardiogram showed hypokinesia of the inferior, inferior-septal and lateral left ventricular walls with an ejection fraction of $40 \%$. On cannulation of the left coronary artery ostium using a 6 French Judkins Left-4 diagnostic catheter, the left circumflex coronary artery (LCx) was found tortuous and totally occluded (Figure 1A). Therefore, 


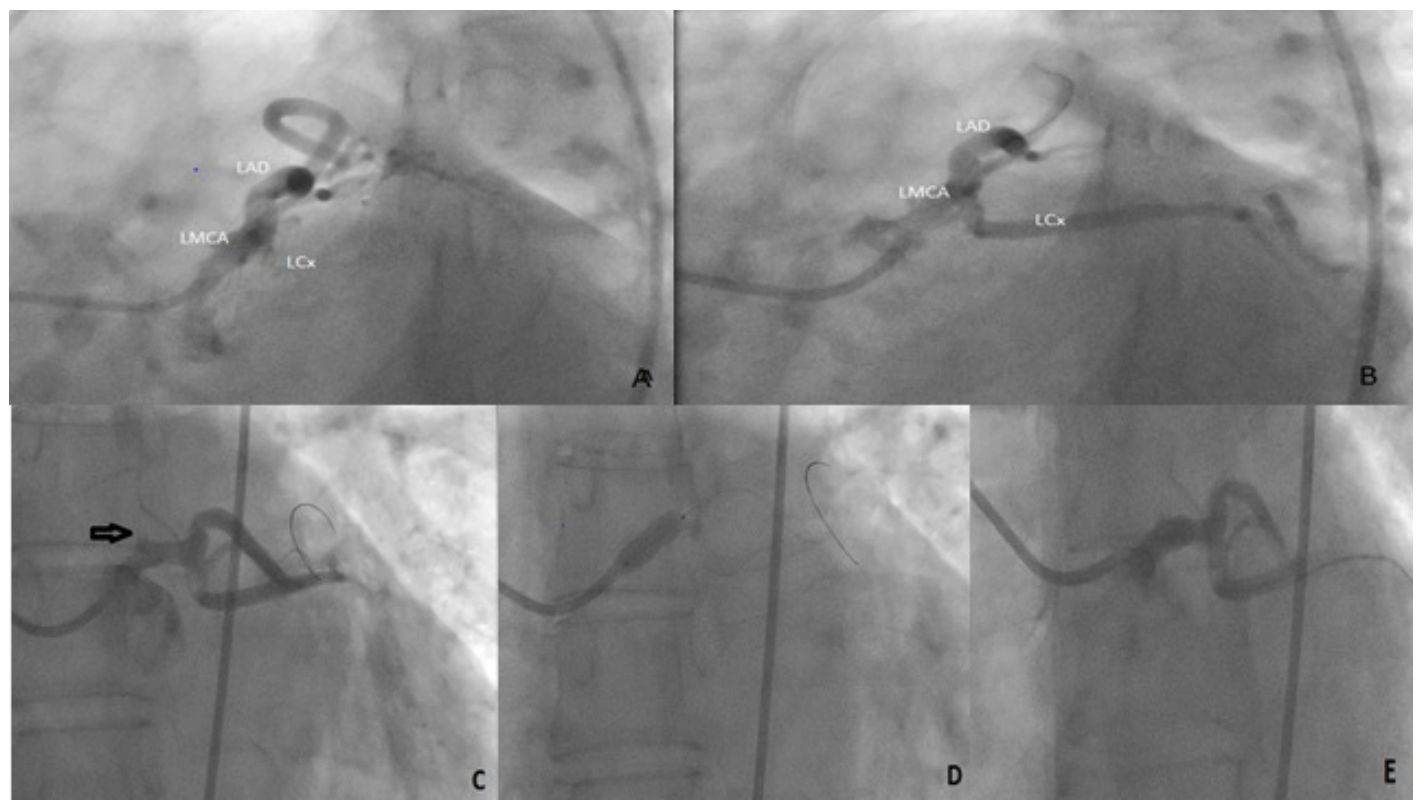

Figure 1. Coronary angiogram images of the lesions during the procedure. (A), Angiogram revealing a completely occluded left circumflex artery; (B), Angiogram showing restoration of thrombolysis in myocardial infarction (TIMI) grade 3 flow in the left circumflex artery after stenting; (C), Left main coronary Artery dissection (arrow), extending to LCx artery in the anteroposterior caudal view; (D), Deployment of a PROMUS $4.5 \times 12 \mathrm{~mm}$ stent into left main coronary artery; (E), Normal angiographic appearance of the left main coronary artery following stent implantation in left anterior oblique caudal view.

we scheduled to perform PCI to the LCx artery. The LMCA was selectively cannulated without difficulty with a 7 French Judkin's Left-4 guiding catheter (Cordis J\&J, Miami, FL). The lesion was crossed by a 0.014 inch soft-tip floppy wire (BMW, Abbott Vascular, Santa Clara, CA, USA) and predilated with $2.0 \times 12$ $\mathrm{mm}$ compliant balloon at $12 \mathrm{~atm}$. After predilation, a $3.0 \times 28 \mathrm{~mm}$ PROMUS (Boston Scientific Co., Natick, MA, USA) DES was uneventfully deployed across the lesion (Figure 1B). Because of stent under expansion at the site of the tight lesion we successfully performed post dilation with use of a $3.5 \times 15 \mathrm{~mm}$ noncompliant balloon. Subsequent angiography revealed persistent contrast staining outside the coronary lumen at the site of the LMCA ostium, which was compatible with a type $\mathrm{C}$ coronary dissection that extended anterogradely to involve the LCx artery (Figure 1C). The patient was pain free and hemodynamically stable without electrocardiographic evidence of ischemia. A $4.5 \times 12$ mm PROMUS (Boston Scientific Co., Natick, MA, USA) DES was deployed at $14 \mathrm{~atm}$ into left main coronary artery (Figure 1D). Post dilation was carried out with a $5.0 \times 10 \mathrm{~mm}$ noncompliant balloon and final angiography showed complete sealing of the LMCA dissection flap (Figure 1E), but residual dissection flap remained between LMCA and LCx artery. Within same day new intervention was established because of a persistent dissection segment (Figure 2). Left main cannulated with extra-support $7 \mathrm{Fr}$., 3.5 EBU coronary-guide catheter (Medtronic Inc., Minneapolis, Minnesota) and floppy guidewire was appropriately located in distal position of LCx artery. A decision was taken to attempt to balloon inflations and stent

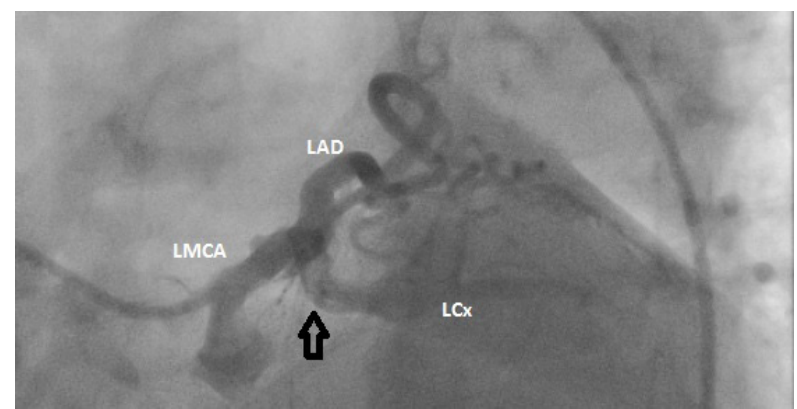

Figure 2. Dissection imaging. Residual dissection between LMCA and LCx viewed in an LAO caudal projection.

deployment at dissected segment, however, multiple attempts failed due to proximal tortuosity of LCx. Deep engagement of guiding catheter compressed the proximal stent struts and resulted in longitudinal stent deformation (Figure 3). Due to deteriorating coronary flow and enlargement of the dissected lumen it was decided to shift the patient for emergency surgical intervention. Successful surgery was performed and patient was discharged from ICU on the 5 th postoperative day without any complications.

\section{Discussion}

Catheter-induced LMCA dissection is a rare but wellrecognized life-threatening complication of coronary angiography and angioplasty. Unusual LMCA anatomy or location, presence of LMCA atherosclerosis, operator experience, vigorous hand injection of contrast medium, selection of catheter type (left Amplatz guiding catheters are associated with a higher risk of 


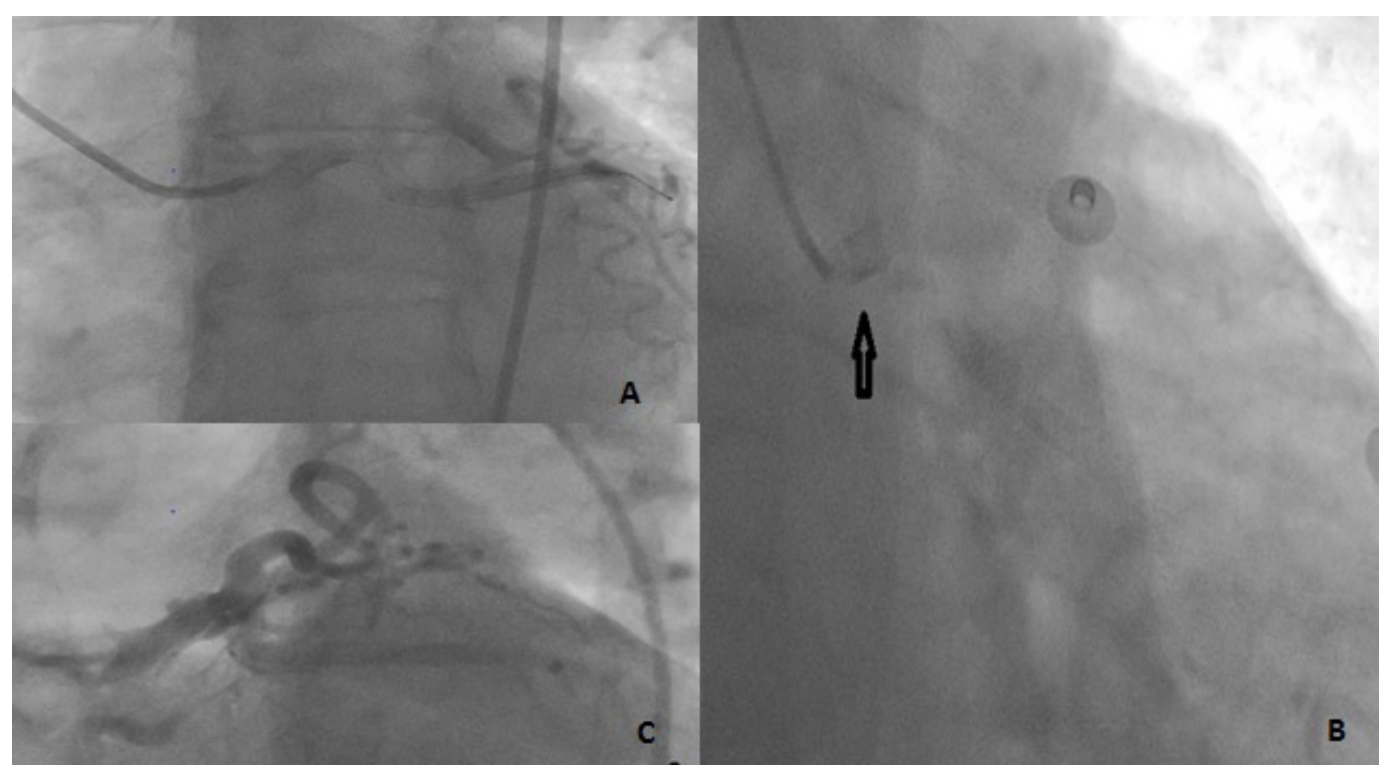

Figure 3.The progression of longitudinal deformation. (A), Noncontrast angiogram after LMCA stent deployment; (B), Longitudinal deformation and shortening of the LMCA stent (black arrow pointing at the proximal deformed edge of the stent); (C), Angiogram revealed progressive enlargement of the dissected lumen and deteriorating coronary flow.

dissection), inappropriate positioning of the catheter in co-axial alignment with LMCA and subintimal passage of the guidewire (especially stiffer and less manageable guide wires) have all been associated with an increased risk of iatrogenic LMCA dissection (5-7). Iatrogenic LMCA dissection is twice as likely to occur during coronary intervention compared to diagnostic angiography (8) and deep engagement of guiding catheters while the angioplasty balloon is withdrawn is often suggested to be responsible for iatrogenic LMCA dissection (1).

LSD has been described as longitudinal distortion or shortening of the stent in the longitudinal axis (4). The literature states that Promus Element stent has commonly been associated with LSD (9). LSD is more frequent in LMCA, ostial, bifurcation and complex lesions, in terms of procedural characteristics, LSD is related to the use of extra-support guiding catheters, extra-support guidewires, use of more than one stent and post-dilatation, and lower stent inflation pressure (10). The treatment of LSD includes dilatation of a deformed segment with appropriate sized noncompliant balloon and if required, another stent for optimal end results Although rare, the LSD can lead to potential complications such as predisposing to stent thrombosis (4). In rare cases, percutaneous management of stent deformation might become difficult, such that, surgery becomes necessary in such situations.

The strategy for managing of LMCA dissection is variable and depends upon the mechanism, the comorbidities of the patient and degree of hemodynamic stability. Hemodynamically unstable patients are most likely to undergo percutaneous coronary intervention (PCI), because PCI (bailout stenting) of LMCA can be performed rapidly shortly after iatrogenic LMCA dissection occurred and has high technical success. Coronary artery bypass grafting $(\mathrm{CABG})$ is particularly valuable in a hemodynamically stable patient with extensive iatrogenic LMCA dissection (antegrade or retrograde extension into the aortic root) and multivessel disease.

In conclusion, we hereby report a case of iatrogenic LMCA dissection in which bailout stenting failed due to LSD. LSD is a rarely encountered complication but can be seen in complex lesions such as ostial, bifurcation and LMCA lesions. To prevent LSD, aggressive catheter manipulation and deep engagement of catheter during withdrawal of stent balloons, post-dilatation balloons or adjunctive imaging devices should be avoided. The interventionists must be aware of this complication and must recognize and manage it carefully.

\section{References}

1. Awadalla H, Sabet S, El Sebaie A, Rosales O, Smalling R. Catheter-induced left main dissection incidence, predisposition and therapeutic strategies experience from two sides of the hemisphere. J Invasive Cardiol. 2005; 17:233-236.

2. Kedhi E, Gomes ME, Lagerqvist B, Smith JG, Omerovic E, James S, Harnek J, Olivecrona GK. Clinical impact of second-generation everolimus-eluting stent compared with first-generation drug-eluting stents in diabetes mellitus patients: Insights from a nationwide coronary intervention register. JACC Cardiovasc Interv. 2012; 5:1141-1149.

3. Planer D, Smits PC, Kereiakes DJ, Kedhi E, Fahy M, Xu K, Serruys PW, Stone GW. Comparison of everolimus- and paclitaxel-eluting stents in patients with acute and stable coronary syndromes: Pooled results from the SPIRIT (a clinical evaluation of the XIENCE V everolimus eluting coronary stent system) 
and COMPARE (a trial of everolimus-eluting stents and paclitaxel-eluting stents for coronary revascularization in daily practice) trials. JACC Cardiovasc Interv. 2011; 4:1104-1115.

4. Hanratty CG, Walsh SJ. Longitudinal compression: A "new" complication with modern coronary stent platforms-time to think beyond deliverability? EuroIntervention. 2011; 7:872-877.

5. Onsea K, Kayaert P, Desmet W, Dubois CL. Iatrogenic left main coronary artery dissection. Neth Heart J. 2011; 19:192-195.

6. Kovac JD, de Bono DP. Cardiac catheter complications related to left main stem disease. Heart. 1996; 76:76-78.

7. Slack JD, Pinkerton CA, VanTassel JW, Orr CM. Left main coronary artery dissection during percutaneous transluminal coronary angioplasty. Cathet Cardiovasc Diagn. 1986; 12:255-260.

8. Eshtehardi P, Adorjan P, Togni M, Tevaearai H, Vogel R,
Seiler C, Meier B, Windecker S, Carrel T, Wenaweser P, Cook S. Iatrogenic left main coronary artery dissection: Incidence, classifcation, management, and long-term follow-up. Am Heart J. 2010; 159:1147-1153.

9. von Birgelen C, Sen H, Lam MK, et al. Third-generation zotarolimus-eluting and everolimus-eluting stents in all-comer patients requiring a percutaneous coronary intervention (DUTCH PEERS): A randomised, singleblind, multicentre, non-inferiority trial. Lancet. 2014; 383:413-423.

10. Guler A, Guler Y, Acar E, Aung SM, Efe SC, Kilicgedik A, Karabay CY, Barutcu S, Tigen MK, Pala S, İzgi A, Esen AM, Kirma C. Clinical, angiographic and procedural characteristics of longitudinal stent deformation. Int $\mathrm{J}$ Cardiovasc Imaging. 2016; 32:1163-1170.

(Received August 1, 2018; Revised August 13, 2018; Accepted August 19, 2018) 